\title{
The Switching Message Estimator for Network-Based Motion Control Systems
}

\author{
Chen-Chou Hsieh and Pau-Lo Hsu \\ Department of Electrical Engineering, National Chiao-Tung University, 1001 Ta Hsueh Road, Hsinchu 300, Taiwan \\ Correspondence should be addressed to Pau-Lo Hsu, plhsu@mail.nctu.edu.tw \\ Received 2 January 2012; Revised 20 March 2012; Accepted 21 March 2012 \\ Academic Editor: Ya-Jun Pan
}

Copyright () 2012 C.-C. Hsieh and P.-L. Hsu. This is an open access article distributed under the Creative Commons Attribution License, which permits unrestricted use, distribution, and reproduction in any medium, provided the original work is properly cited.

\begin{abstract}
Missing commands from the interpolator caused by the dropout effect of network transmission will cause motion error in motion plants implemented on network-based control systems (NCSs). Dropout data can be properly recovered by applying different message estimators to improve motion contouring accuracy. This study shows that the dropout rate and the distribution of missing commands dominate the motion error, and that more centralized missing commands result in a higher maximum contouring error. The short-window dropout quantity (SDQ) is proposed in this paper to estimate the network quality based on the dropout rate and its distribution of the missing data. Furthermore, according to the condition of missing data based on the SDQ, the switching least-square estimator (LSE) is proposed to compensate for missing motion commands. Simulation and experimental results on the two-axis AC servo motor NCS indicate that motion contouring accuracy is greatly improved by applying the proposed estimator.
\end{abstract}

\section{Introduction}

Recently, network-based control systems (NCSs) have been widely studied because of their advantages, such as lower cost, easier troubleshooting, and implementation flexibility $[1,2]$. However, network-introduced time delay is unavoidable and the data dropout of NCS becomes significant as node and data length increase or as the system sampling time decreases with a limited network bandwidth. Recently, coping with the network-introduced delay on NCS has been widely studied using various approaches, such as robust $H_{\infty}$ control [3], passive control [4], and predictive control [5]. These methods handle the stochastic network delay with the assumption that the delay is either relatively small or similar to the sampling time in NCS.

In real applications, however, the network-induced time delay may be significant enough to cause data traffic congestion and collision; thus, data dropout occurs and leads to the severe degradation of performance in NCS [7]. The linear quadratic Gaussian (LQG) control has been successfully used to solve random packet loss (e.g., TCP-like protocols) to compensate for the missing data in NCS under a relatively low dropout rate [8]. Optimal filtering based on the $H_{n}$-norm estimation error has been presented to handle multiple packet dropouts $[9,10]$. The network predictor control (NPC), which consists of a control prediction generator (GPC), observer-based output predictor (OP), and network delay compensator (NDC), has been proposed to overcome network delay and data dropout rate [11]. A reliable estimator to restore the missing data in real motion NCS applications is still being pursued by automation and industry engineers.

The general NCS architecture in Figure 1 shows that all control and feedback signals are communicated through the network for multiaxis motion systems. However, as the number of motion axes increases, network traffic also increases. Therefore, in real control applications, the practical motion NCS architecture with more efficient transmission for multiaxis system is generally modified, as shown in Figure 2. Only command messages are transmitted from the master to the controller. These control and feedback signals are not sent through the network in multiaxis motion systems. Therefore, the transmission can meet the hard real-time requirement within a sampling period to avoid heavy traffic in networks. 


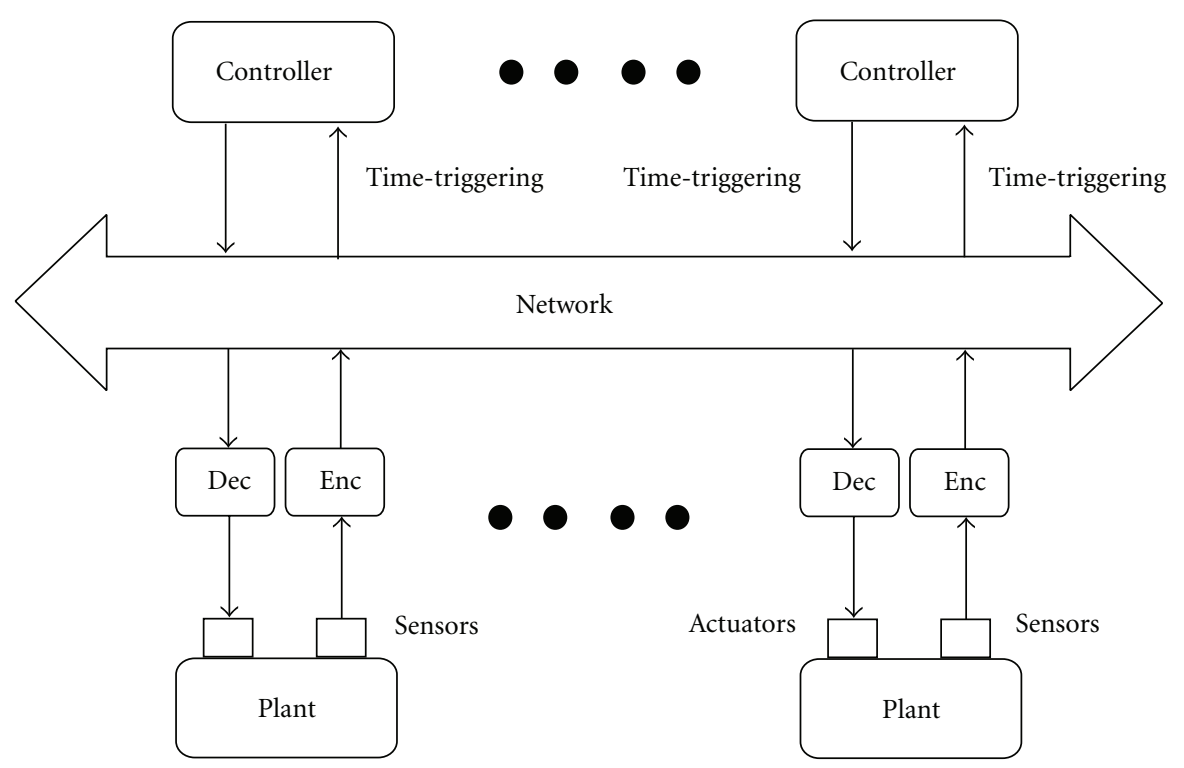

FIGURE 1: The general NCS architecture with multiple nodes.

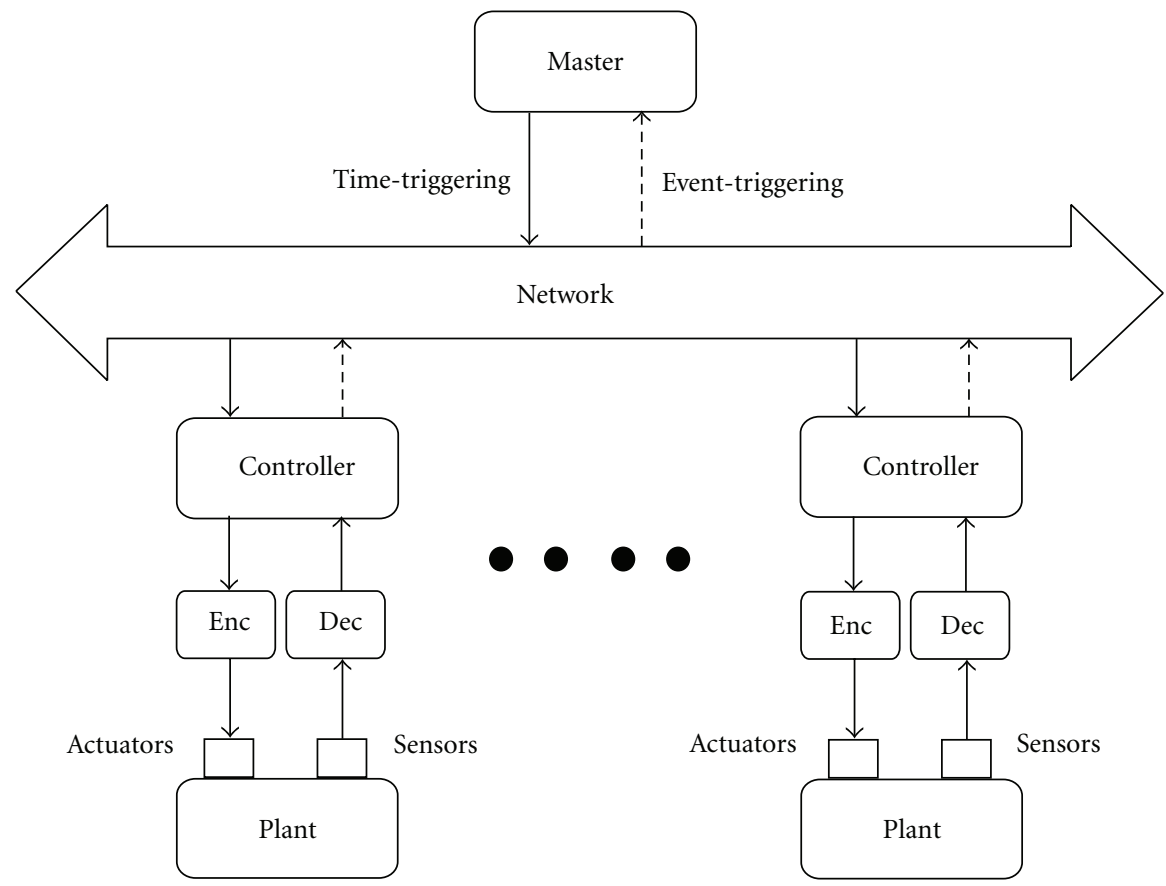

FIgURE 2: The modified NCS architecture with multiaxis motion control systems.

Feedback messages may still be transmitted according to monitoring functions by the event-triggering approach.

However, missing messages in motion NCS become unavoidable when the network delay is longer than the sampling period. Under such circumstances, a message estimator is required to estimate the missing commands and to compensate for their effect in motion accuracy. Various message estimators have been proposed to cope with the dropout effect for motion NCS under different conditions. The 1-delay message estimator is implemented by estimating the missing message using previously received data [12]. The nonlinear NCS has been modeled as a Markovian jump linear system, and the finite loss history estimator (FLHE) has been proposed to improve data dropout effects when the dropout rate is accurately known [13]. However, these methods require an accurate plant/network model. Recently, modelfree strategies for control packet dropout compensators, such as the proportional plus derivative (PD) predictor with a different order of derivatives, have been proposed [14]. The Taylor estimator has also been proposed to significantly improve the control performance of motion control NCS $[6,15]$. These methods are preferred as dropout data over 


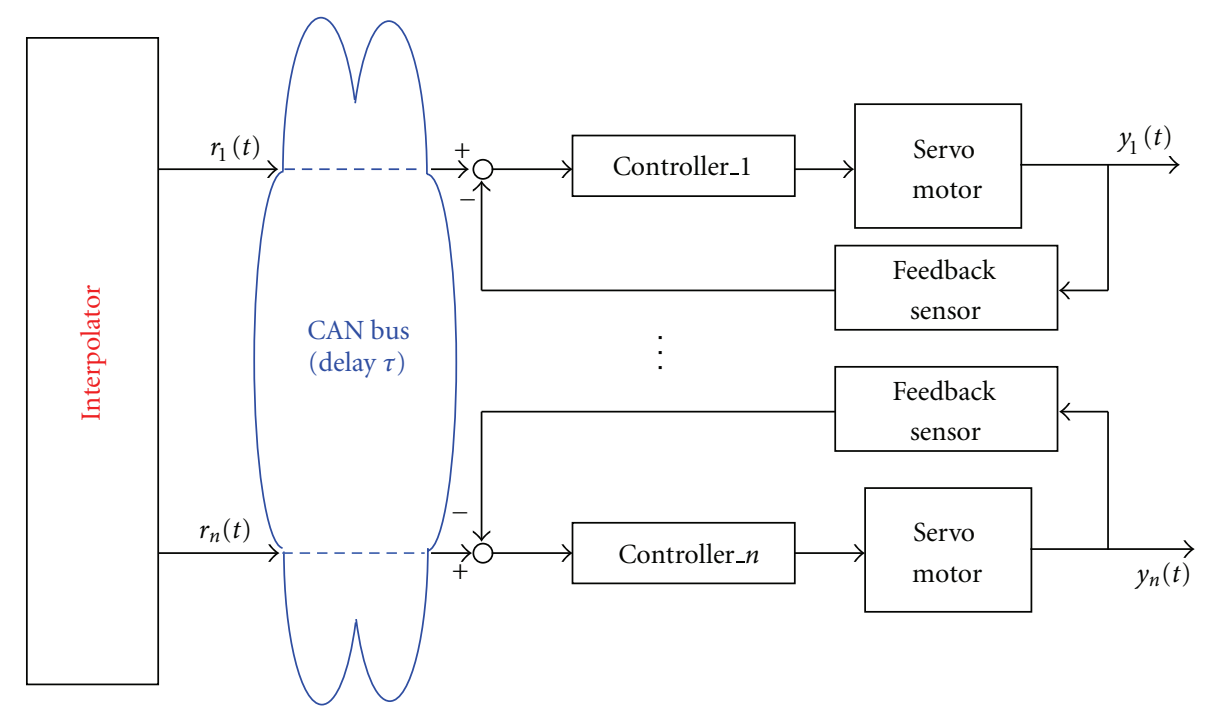

FIgURE 3: The multiaxis motion NCS.

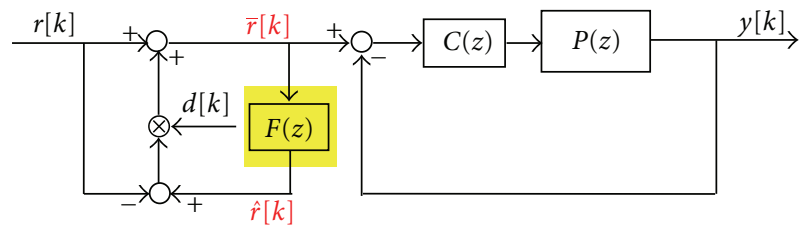

FIgURE 4: The motion NCS with the message estimator [6].

the network are evenly distributed. Missing data that occur in a continuous format tend to lead to a more significant maximum contouring error. Therefore, motion NCS require a suitable index to express the data dropout quantity and to imply its distribution.

This paper proposes the use of short-window dropout quantities (SDQs) to indicate the network communication quality for motion NCS based on both the dropout rate and distribution of the missing data. This paper also proposes an intelligent message estimator (IME) with a switching mechanism based on real-time measured SDQ to obtain a significantly-reduced motion error compared with the 1delay and Taylor message estimators in the literature $[6,12-$ 15].

Data dropout also leads to the problem of asynchronization among different axes. Both simulation and experimental results, with the nonuniform rational B-spline (NURBS) motion commands $[16,17]$, have been verified using the proposed message estimator. Results indicate that the proposed estimator maintains the lowest transmission error and the least motion contouring error as missing messages become more severe. The CAN-based two-axis AC servo motor control system has also been implemented with the proposed estimator. Although a high dropout rate degrades NCS performance, the contouring error in the motion control is also closely related to centralized missing commands. Therefore, an effective approach to estimate the quantity of network communication by considering both the dropout rate and the distribution of missing motion commands is crucial to obtain a reliable motion NCS.

\section{Data Dropout Quantity in Motion NCS}

In motion NCS, the control messages for each motion axis must be transmitted on time through the network protocol to meet control design specifications, as shown in Figure 3. Since the network-induced time delay exists in stochastic and time-varying natures, the transmitted messages may miss the hard real-time deadline because of the limited network bandwidth and the missing data thus occurs.

2.1. Modeling of the Data Dropout. Concerning the missing data in motion NCS, $d$ represents a binary process with probability $P(d[k]=1)=\varepsilon$ and $P(d[k]=0)=1-\varepsilon$, and the data dropout occurs when $d[k]=1$, where $C(z)$ and $P(z)$ represent the controller and the plant, respectively, as shown in Figure 4 [6]. During the network transmission, the general message estimator $F(z)$ is activated to compensate for the lost data packets with the estimated motion commands $\hat{r}$ as [9]:

$$
\begin{array}{ll}
\bar{r}[k]=r[k], & \text { if } d[k]=0, \\
\bar{r}[k]=\hat{r}[k], & \text { if } d[k]=1, \Leftarrow \text { dropout. }
\end{array}
$$

2.2. The Distribution Effect of Data Dropout. Traditionally, the data dropout rate $\varepsilon$ is recognized as the quality of service 


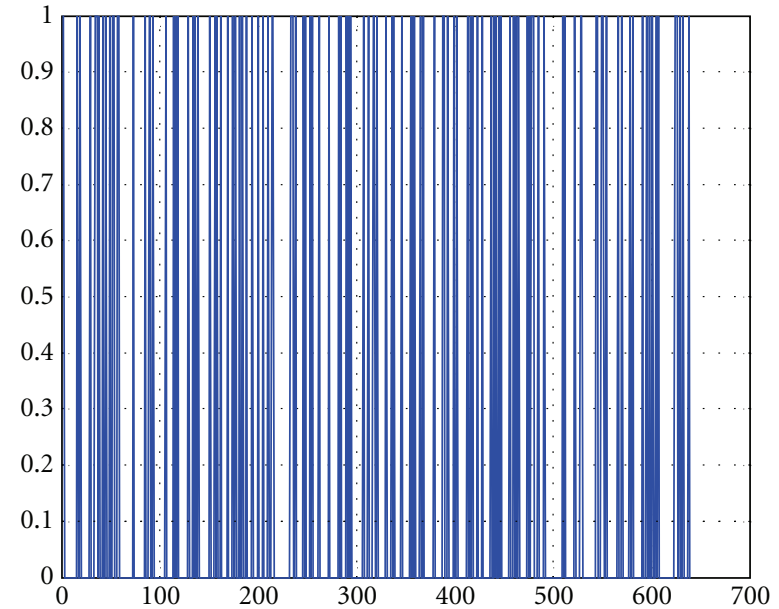

(a)

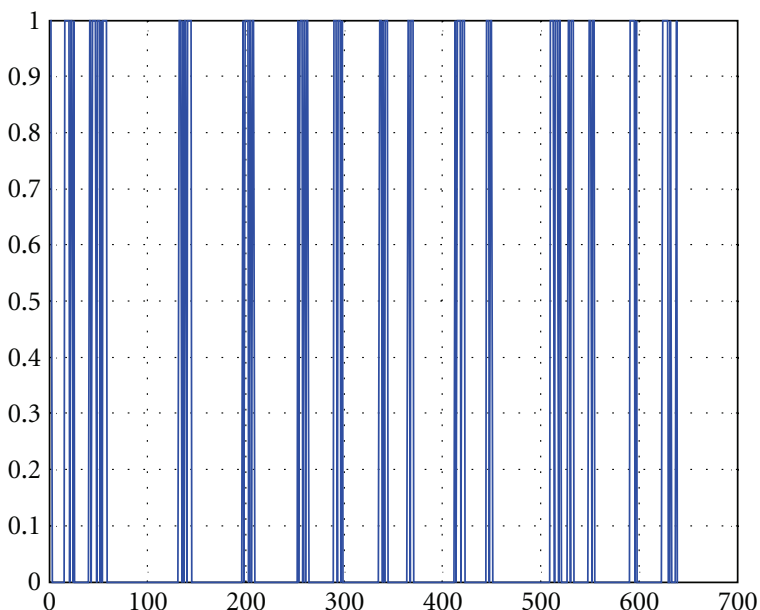

(b)

FIgURE 5: (a) Distributed-dropout signals and (b) centralized-dropout signals with the same dropout rate $20 \%$.

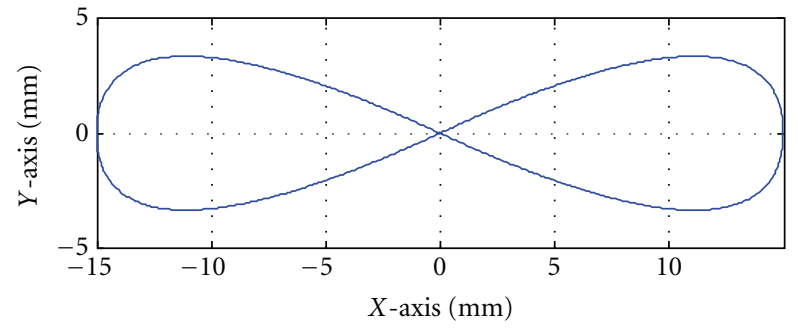

FIgURE 6: NURBS position commands.

(QoS) for most NCSs. However, in motion NCS, centralized missing data will cause a more serious motion error compared with evenly distributed missing data, as shown in Figure 5. Note that Figures 5(a) and 5(b) show two signals with the same data dropout rate of $20 \%$ applied to the butterfly profile for the fifth-order NURBS commands, as shown in Figures 6-7 [6]. By applying the same secondorder Taylor estimator for the missing motion commands, simulation results show that the transmission error is more significant when the data dropout is more centralized, as shown in Figure 7(b). These results indicate that both data dropout and its distribution should be evaluated together particularly applied in motion NCS.

2.3. The SDQ Index. The dropout data of different network infrastructures possess different distributions [18]. It is mentioned before that the distribution of data dropout in NCS is a crucial factor to determine motion accuracy as well as the data dropout. This paper proposes the shortwindows dropout quantities (SDQ) for on-line evaluating the transmission performance of motion NCS. The SDQ is activated only when a missing data occurs, and the total missing data in the past short window is accumulated as in the following:

$$
\operatorname{SDQ} \begin{cases}\text { nonactivated, } & \text { if } d[k]=0, \\ \text { activated, } & \text { if } d[k]=1 .\end{cases}
$$

For example, if the data length of the short window is determined as 5 , six possible receiving/missing states, $0 \sim 5$, is recorded in a buffer during the network communication to indicate its micro QoS. The average of the total SDQ can be recognized and the macro-QoS of the NCS.

2.4. An Illustrative Example of the SDQ. Examples illustrated with three missing data among eighteen transmitted messages with different distribution as shown in Figures 8(a) and $8(\mathrm{~b})$, separately. Even their data dropout rates are the same as $3 / 16$, their average SDQ are different, and the more distributed missing data series presents a lower average value of SDQ as shown in Figure 8(b).

As shown in Figures 5(a) and 5(b) with the same data dropout rate of $20 \%$, their average values of SDQ are very different as 0.9 and 2.1, for more distributed and more centralized dropouts, respectively. Simulation results indicate that as the 3 rd-order Taylor estimator is applied, the contouring error increases when the averaging SDQ increases even with the same data dropout rate, as shown in Figures 6-7. Results also indicate that the index of SDQ is more appropriate to imply the distribution of the dropout data in motion NCS.

\section{The Switching Least-Square Estimator}

In real motion NCS, the position commands are generally in the form of smooth curves. At the curvature varying significantly along the contour, and missing data with higher 


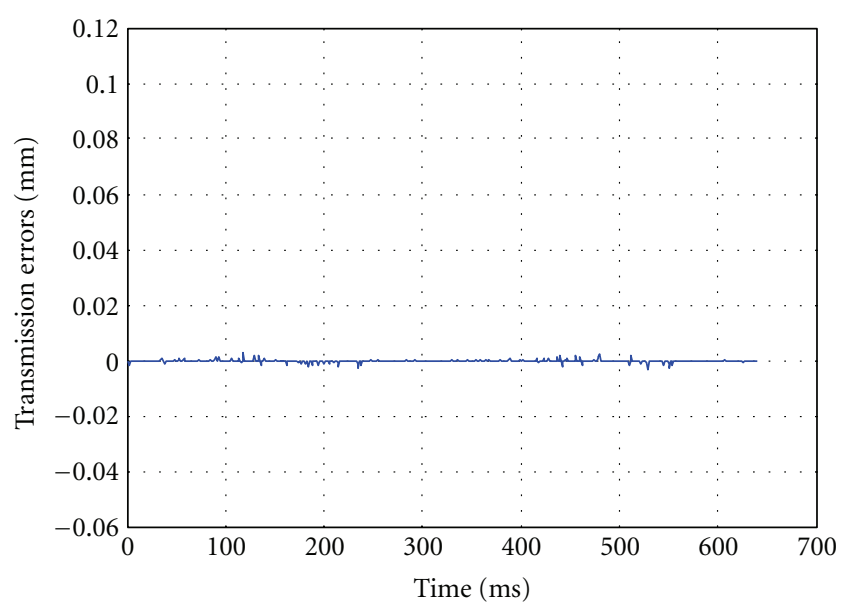

(a)

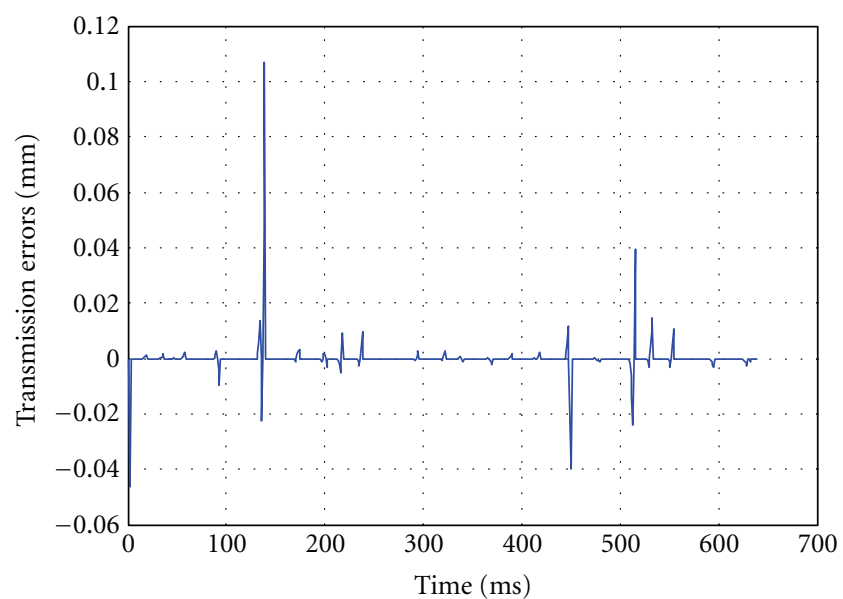

(b)

FIgURE 7: $y$-axis racking errors due to (a) distributed and (b) centralized missing data (20\% dropout rate).

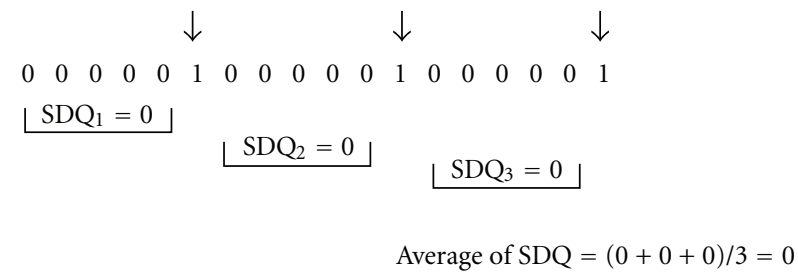

(a)

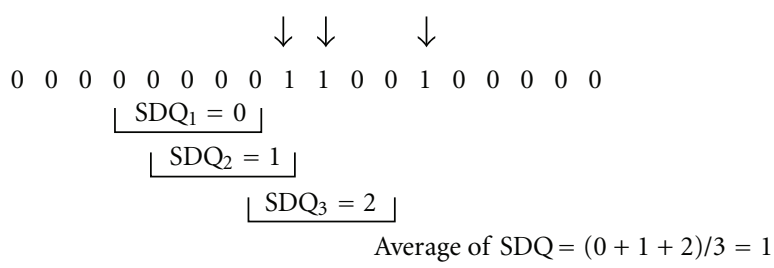

(b)

FIGURE 8: Real-time recorded SDQ with the same dropout rate 3/16 (a) distributed dropout and (b) concentrated dropout.

SDQ generally causes a more serious contouring error. To estimate the missing messages in NCS, the basic 1-delay estimator simply adopts the last received message as the current missing message. Moreover, the Taylor estimator was proposed to estimate the current missing message from past several received signals [15]. However, if the past received signal is also missing, the estimated message by the Taylor estimators thus becomes unreliable.

In this paper, the estimator with a switching mechanism is proposed based on the online measured SDQ with the suitable order of the least-square estimator (LSE). However, as the messages dropout becomes more serious, estimation based on the previous data is no longer reliable and the 1delay estimator will be adopted.

3.1. The Least-Square Estimator. Since the online estimation processing is time consuming and the time-trigger NCS commands are simply in a time series, all parameters of the real-time least-square estimation (LSE) can be obtained in advance. Thus, an online estimation and compensation algorithm for the missing messages in motion NCS are proposed by applying the least-square approach on the past data within a short window. For a general time sequence $x[1], x[2], \ldots, x[M]$, a polynomial sequence can be suitably described as

$$
x[k]=c_{0}+c_{1} k+c_{2} k^{2}+\cdots+c_{N} k^{N} .
$$

Thus,

$$
\begin{gathered}
x[1]=c_{0}+c_{1}+c_{2}+\cdots+c_{N}, \\
x[2]=c_{0}+c_{1} 2+c_{2} 2^{2}+\cdots+c_{N} 2^{N}, \\
\vdots \\
x[M]=c_{0}+c_{1} M+c_{2} M^{2}+\cdots+c_{N} M^{N} .
\end{gathered}
$$

By rearranging (4) as

$$
\left[\begin{array}{c}
x[1] \\
x[2] \\
\vdots \\
x[M]
\end{array}\right]=\left[\begin{array}{cccc}
1 & 1 & \cdots & 1 \\
2^{0} & 2 & \cdots & 2^{N} \\
\vdots & \vdots & & \vdots \\
M^{0} & M & \cdots & M^{N}
\end{array}\right]\left[\begin{array}{c}
c_{0} \\
c_{1} \\
\vdots \\
c_{N}
\end{array}\right] \equiv x=A \cdot c .
$$

The normal equation from the least-square approach can be applied to obtain the coefficient vector $c$ as

$$
c=\left(A^{T} A\right)^{-1} A^{T} x .
$$




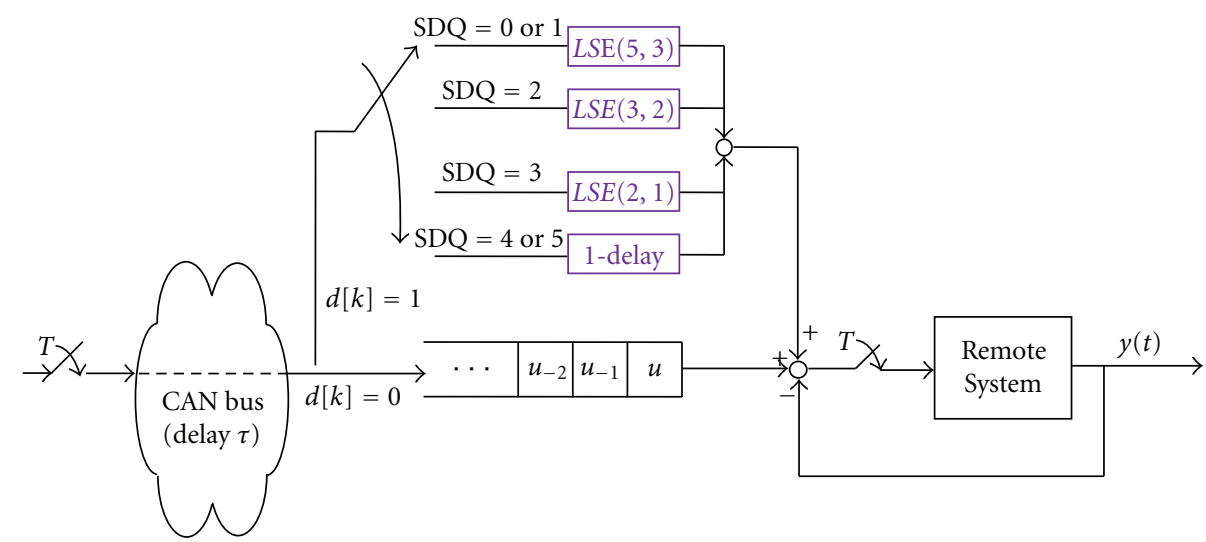

FIGURE 9: The architecture of the proposed estimator with a switching mechanism.

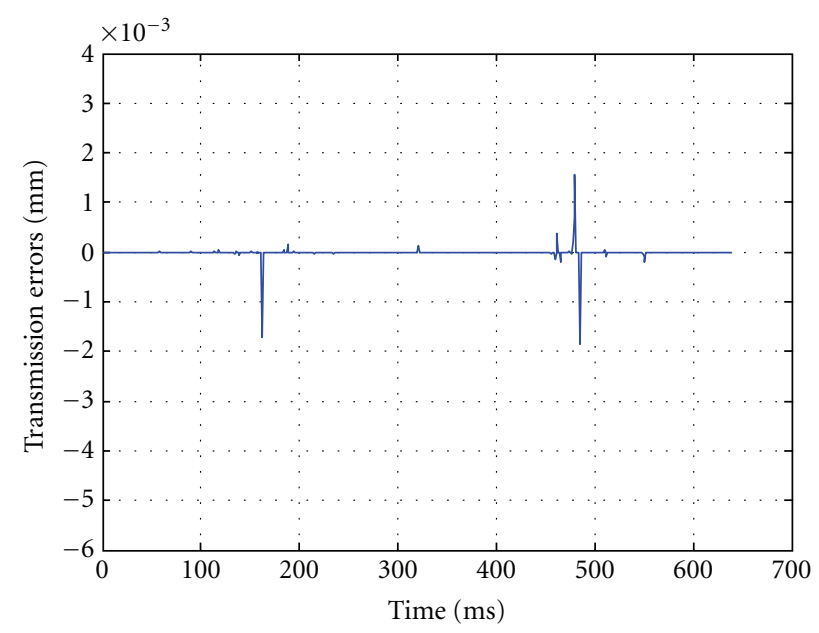

(a)

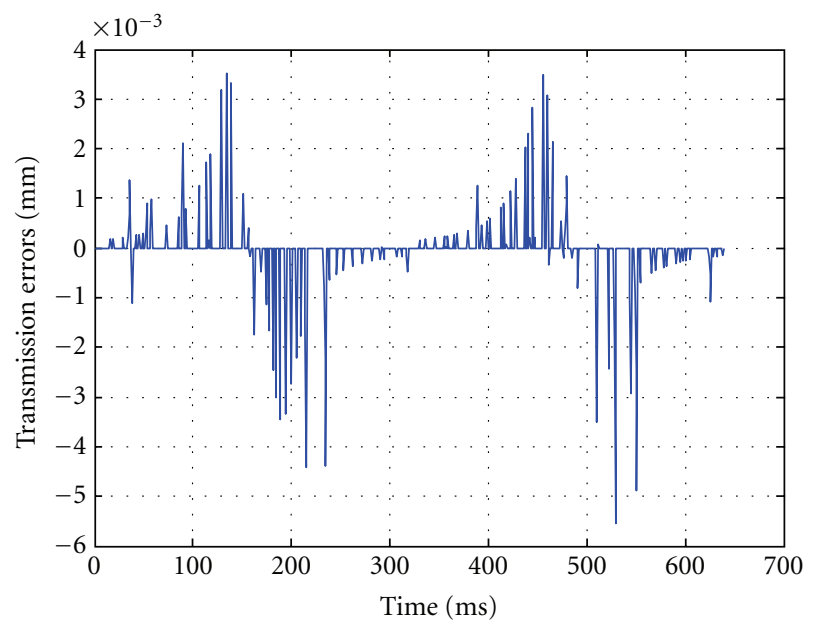

(b)

FIgure 10: Transmission errors with (a) $\operatorname{LSE}(5,3)$ and (b) $\operatorname{LSE}(2,1)$ with the average SDQ $=0.9$.

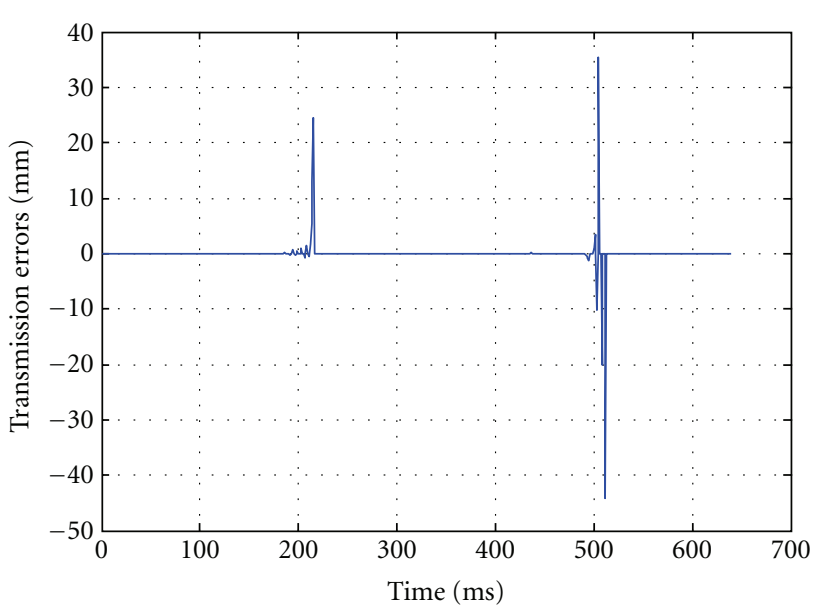

(a)

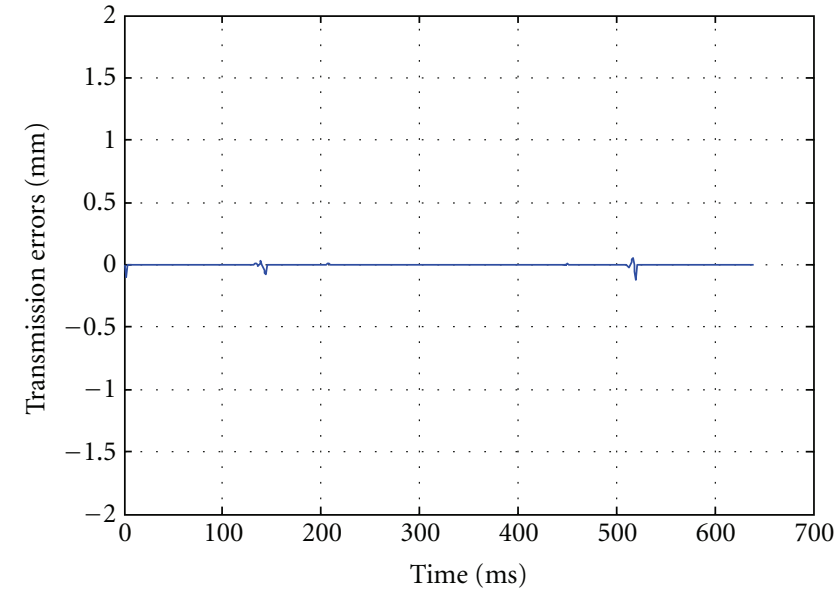

(b)

FIgURE 11: Transmission errors with (a) $\operatorname{LSE}(5,3)$ and $(b) \operatorname{LSE}(2,1)$ with the average SDQ $=1.9$. 


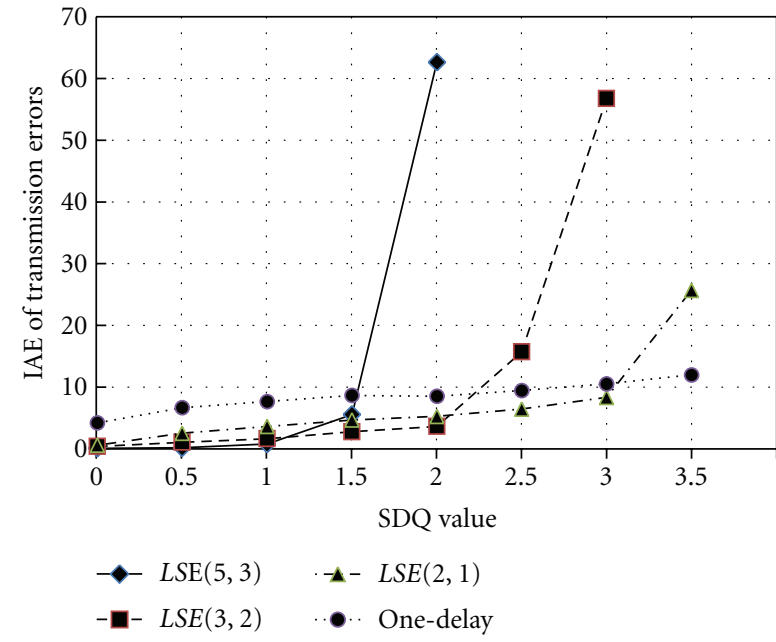

FIGURE 12: Analysis of compensation effects with averaging SDQ.

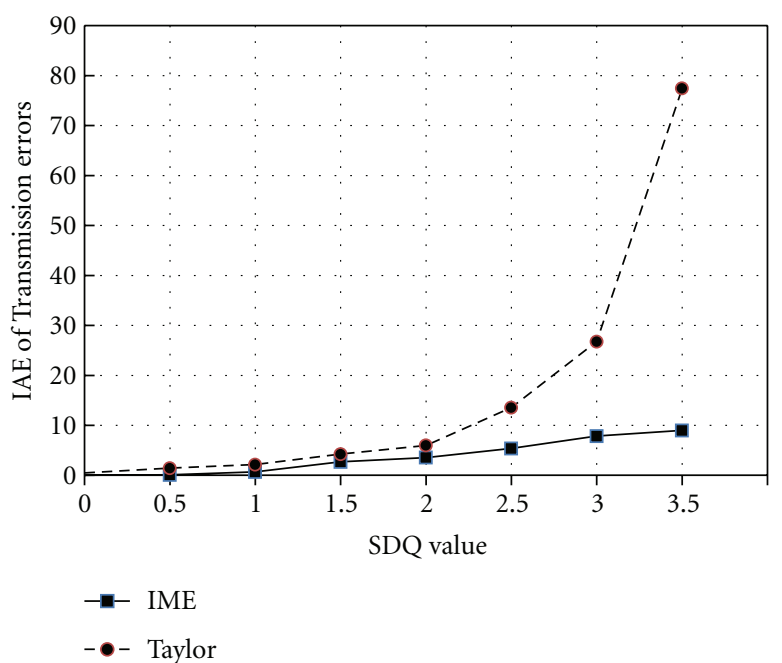

FIgURE 13: Simulation results with different estimators.

Thus, the missing value can be estimated based on the past $M$ signals with the $N$ th order of the least-square estimator as

$$
\begin{aligned}
& x[M+1]=c_{0}+c_{1}(M+1)+c_{2}(M+1)^{2}+\cdots \\
& +c_{N}(M+1)^{N} \\
& =\left[\begin{array}{llll}
(M+1)^{0} & (M+1)^{1} & \cdots & (M+1)^{N}
\end{array}\right] \cdot c \\
& =\left[\begin{array}{llll}
(M+1)^{0} & (M+1)^{1} & \cdots & (M+1)^{N}
\end{array}\right] \\
& \text { - }\left(A^{T} A\right)^{-1} A^{T} x \\
& \equiv \operatorname{LSE}(M, N) \cdot x \text {, }
\end{aligned}
$$

and the estimator matrix $\operatorname{LSE}(M, N)$ can thus be preobtained for real-time implementation, where $M$ also indicates the data number in a window to be counted and $N$ is the order of polynomial functions with $N+1$ coefficients.
3.2. Coefficients of the Least-Square Estimators. To achieve an online estimation for motion NCS, the order and the number of data within the window of the least-square estimator should be determined based on the motion commands. For example, the NURBS signal can be approximated by a thirdorder polynomial equation obtained from the LSE [19]. Practically, the window length can be properly chosen as large as five to estimate the missing NURBS commands or other motion contours. In practice, three useful $\operatorname{LSE}(M, N)$ are precalculated for real-time applications based on the quality of communication of NCS as follows.

3.2.1. LSE (5,3) for Low Dropout Cases. The third-order $\operatorname{LSE}(5,3)$ can properly estimate the motion trajectory concerning its position, velocity, acceleration, and even the change of acceleration as the jerk. In this transmission case, all data within the window length 5 are properly received, or at most, only one missing data is estimated within the window. $\operatorname{LSE}(5,3)$ is chosen to estimate a cubic-curve motion command with the order of 3 by using all five previous data, which may include at most one estimated data. The parameters of $\operatorname{LSE}(5,3)$ are determined from (5) as follows:

$$
A=\left[\begin{array}{cccc}
1 & 1 & 1 & 1 \\
1 & 2 & 4 & 8 \\
1 & 3 & 9 & 27 \\
1 & 4 & 16 & 64 \\
1 & 5 & 25 & 125
\end{array}\right],
$$

and by (6)-(7),

$\left[\begin{array}{llll}1 & 6 & 36 & 216\end{array}\right]\left(A^{T} A\right)^{-1} A^{T}=\left[\begin{array}{lllll}-0.8 & 2.2 & -0.8 & -2.8 & 3.2\end{array}\right]$.

The $\operatorname{LSE}(5,3)$ is thus obtained as

$$
\operatorname{LSE}(5,3)=3.2 z^{-1}-2.8 z^{-2}-0.8 z^{-3}+2.2 z^{-4}-0.8 z^{-5} .
$$

3.2.2. $\operatorname{LSE}(3,2)$ for Medium Dropout Cases. In this case, the medium data dropout condition occurs, and the missing data within the short window length 5 are as large as 2 . In other words, at most only three reliable data are accountable within the window to correctly estimate the missing data. Therefore, $\operatorname{LSE}(3,2)$ is chosen to estimate the quadric-curve trajectory with the order of 2 by using three previous data through considering both its position, velocity and acceleration from (7) as

$$
\operatorname{LSE}(3,2)=3 z^{-1}-3 z^{-2}+z^{-3} .
$$

3.2.3. $\operatorname{LSE}(2,1)$ for Heavy Dropout Cases. In this situation, only two data within the window are received, and $\operatorname{LSE}(2,1)$ is chosen to estimate the motion trajectory concerning as high as its velocity only by applying previous two data as

$$
\operatorname{LSE}(2,1)=2 z^{-1}-z^{-2} \text {. }
$$




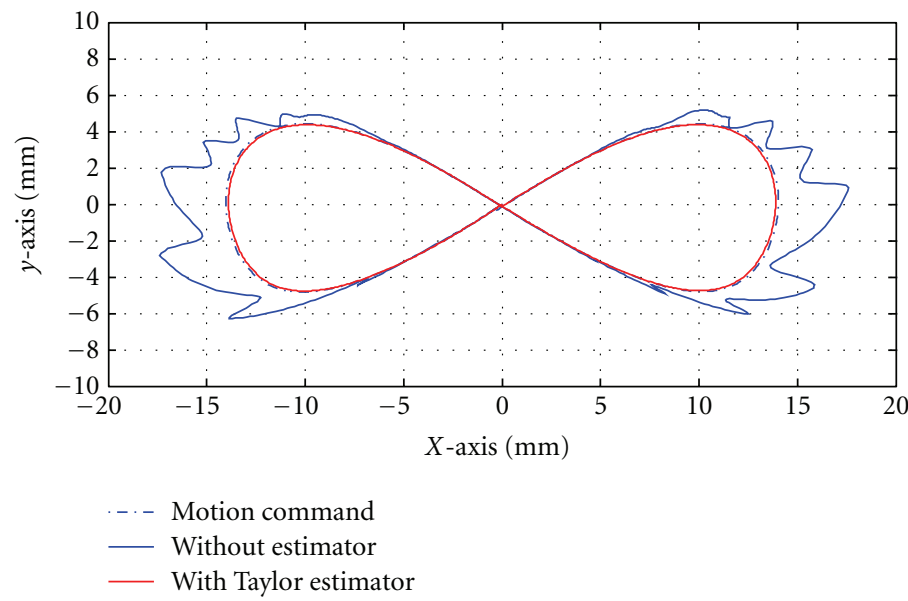

FIGURE 14: Contouring of motion NCS without/with the Taylor estimator (average SDQ $=0.8$ ).

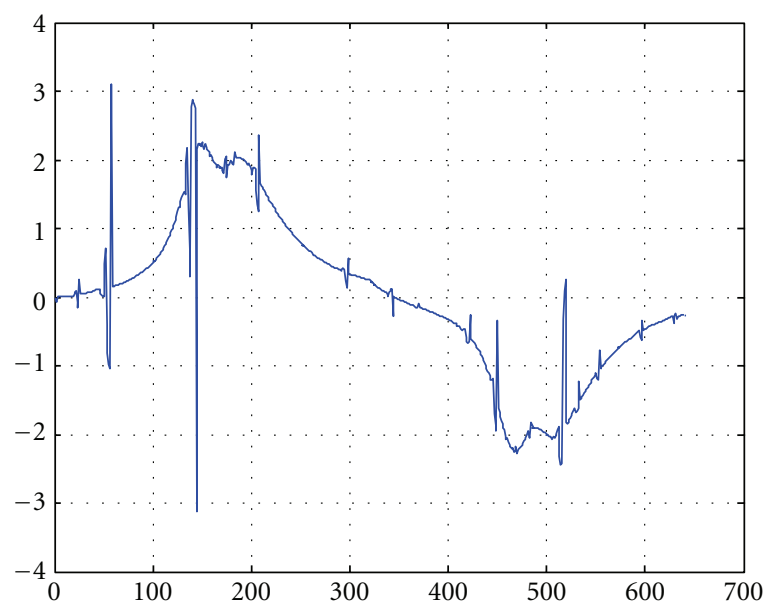

(a)

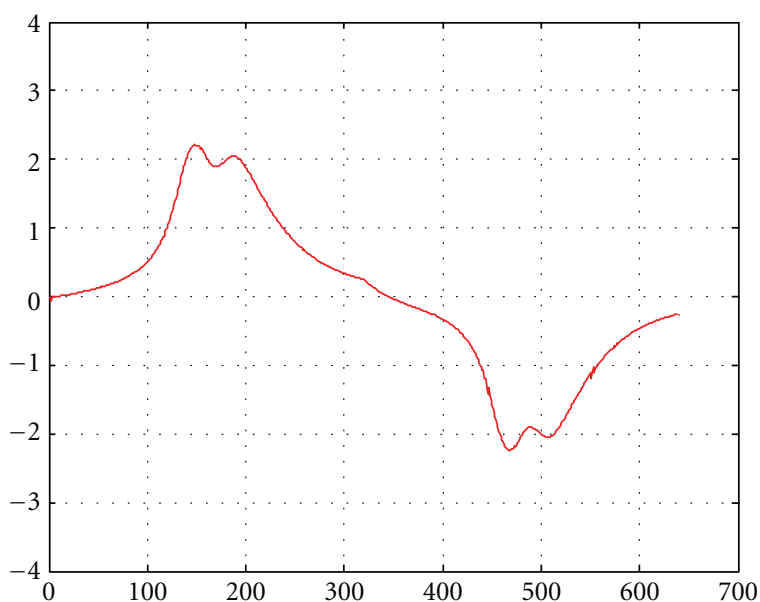

(b)

FIGURE 15: Contouring errors with (a) the Taylor estimator (b) IME (average SDQ =0.8).

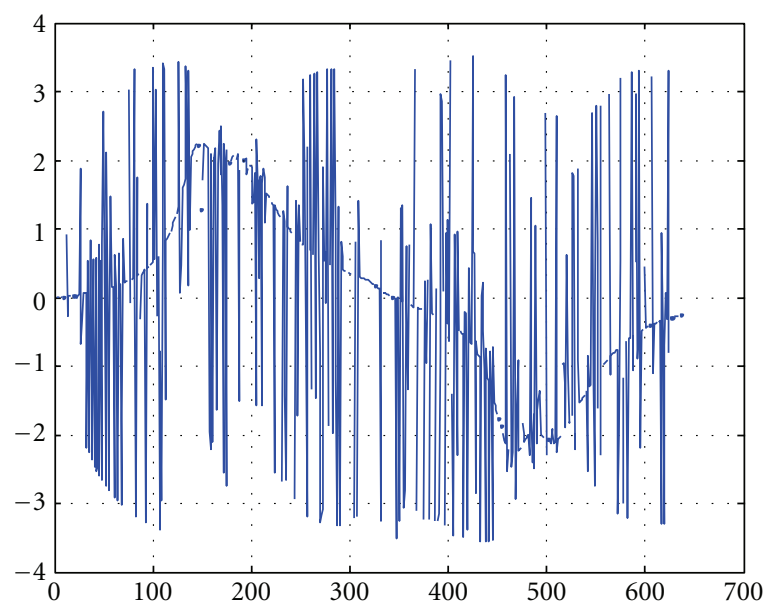

(a)

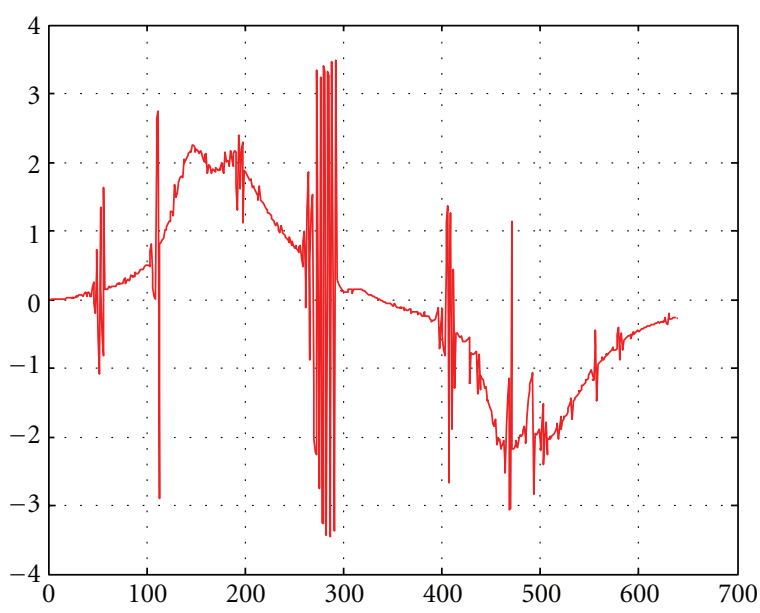

(b)

FIgURE 16: Contouring errors with (a) the Taylor estimator and (b) IME (average SDQ = 2.6). 


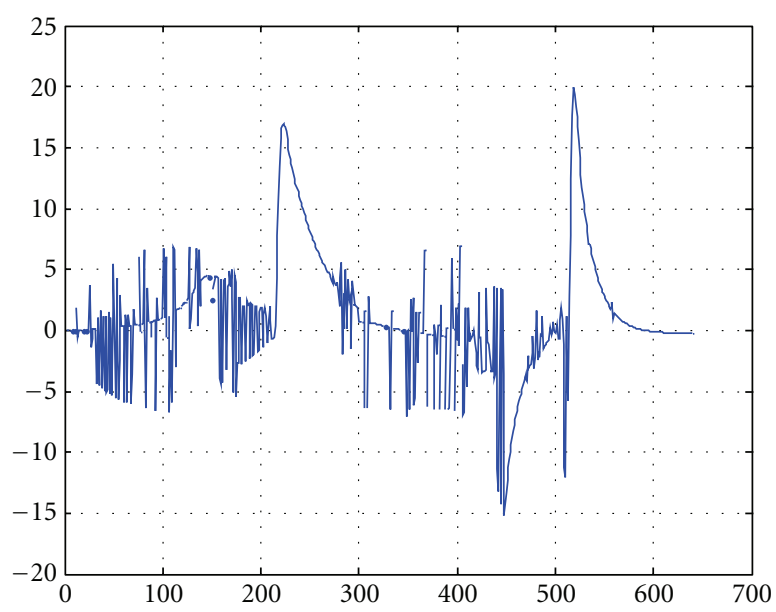

(a)

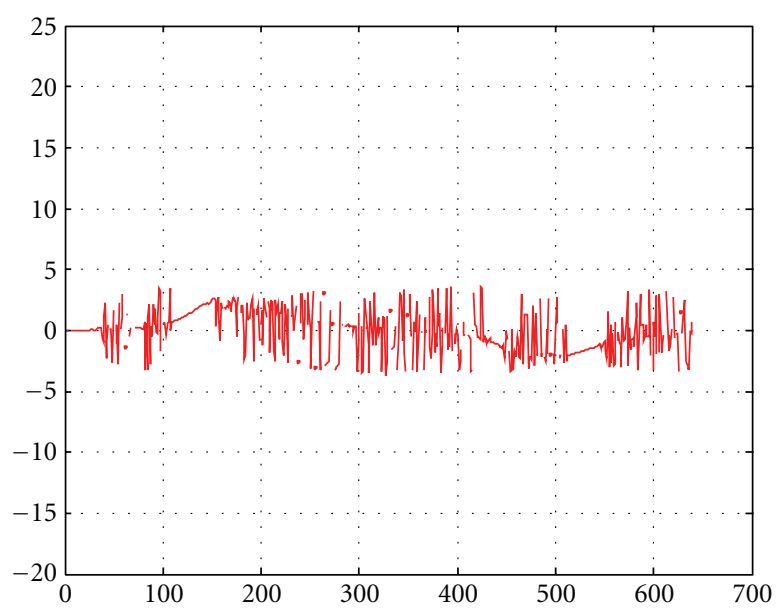

(b)

FIGURE 17: Contouring errors with (a) the Taylor estimator and (b) IME (average SDQ = 3.1).

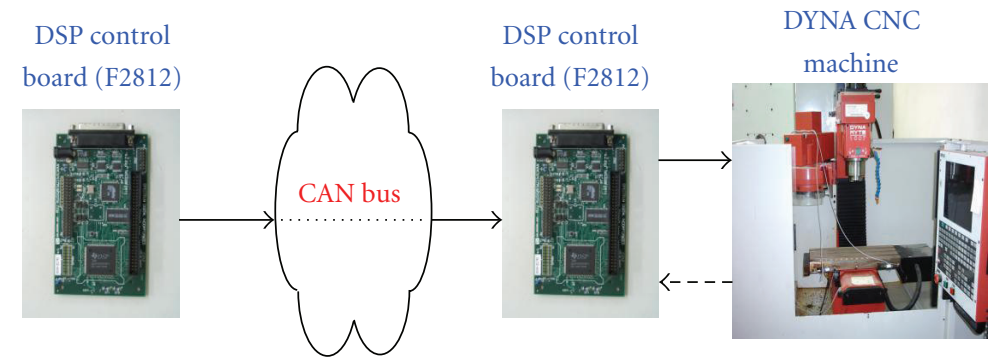

Figure 18: Experimental setup.

3.2.4. The 1-Delay Estimator for Serious Dropout Cases. In this situation, network communication presents such a heavy data dropout rate, either only one data is received within the previous window of length 5 , or all five previous adopted data are obtained through the estimation process. Therefore, estimation results based on the least-square approach by adopting all data within the window length 5 is not reliable anymore, and the 1-delay estimator is determined under such circumstance to estimate the position only by directly adopting the previous data as $[6,12]$

$$
\text { 1-delay estimator }=z^{-1} \text {. }
$$

In summary, different estimators are applied to different transmission conditions based on the SDQ, as shown in Figure 9 as:

$$
\operatorname{SDQ}=\left\{\begin{array}{lr}
0 \text { or } 1, & \operatorname{LSE}(5,3) \text { is adopted, } \\
2, & \operatorname{LSE}(3,2) \text { is adopted } \\
3, & \operatorname{LSE}(2,1) \text { is adopted } \\
4 \text { or } 5, & \text { 1-delay estimator is adopted. }
\end{array}\right.
$$

\section{Analysis of the SDQ on Motion NSC}

Figure 10 shows the transmission error obtained by applying the estimators of $\operatorname{LSE}(5,3)$ and $\operatorname{LSE}(2,1)$ to the motion NCS as the simulation shown in Figure 7. The case of SDQ $=0.9$ implies that one data (close to 0.9 in average) is missing in average within the window length 5. Simulation results show that $\operatorname{LSE}(5,3)$ renders a better compensation effect as compared to $\operatorname{LSE}(2,1)$ which should be applied to a more serious data dropout case. However, as the SDQ increases to 1.9, of which implies that there are about two missing messages among the five transmitted messages, the transmission error increases and $\operatorname{LSE}(5,3)$ is not suitable anymore. Figure 11 shows that the compensation results applying $\operatorname{LSE}(2,1)$ render better performance.

Furthermore, the least-square approach with a different $M$ applied to a different averaging SDQ shows that applying $\operatorname{LSE}(5,3)$ to compensate for the low missing data rate presents the best motion accuracy as the averaging SDQ is less than one, but it becomes the worst as the averaging SDQ increases larger than 1.5, as shown in Figure 12. Moreover, $\operatorname{LSE}(3,2)$ is more suitable for the situation as the averaging SDQ is between 1 and 2 . Note that $\operatorname{LSE}(2,1)$ is most suitable for the situation as the averaging SDQ is between 


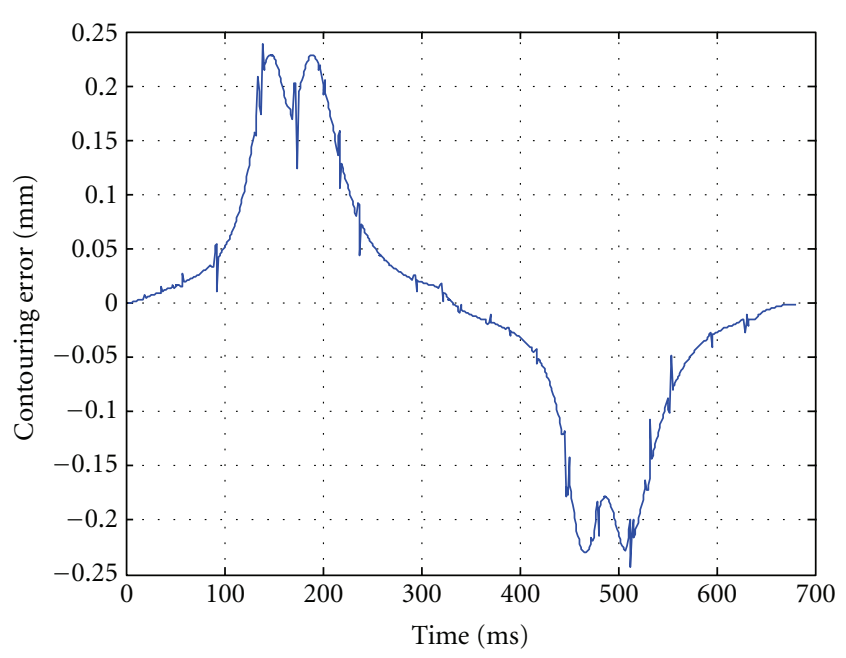

(a)

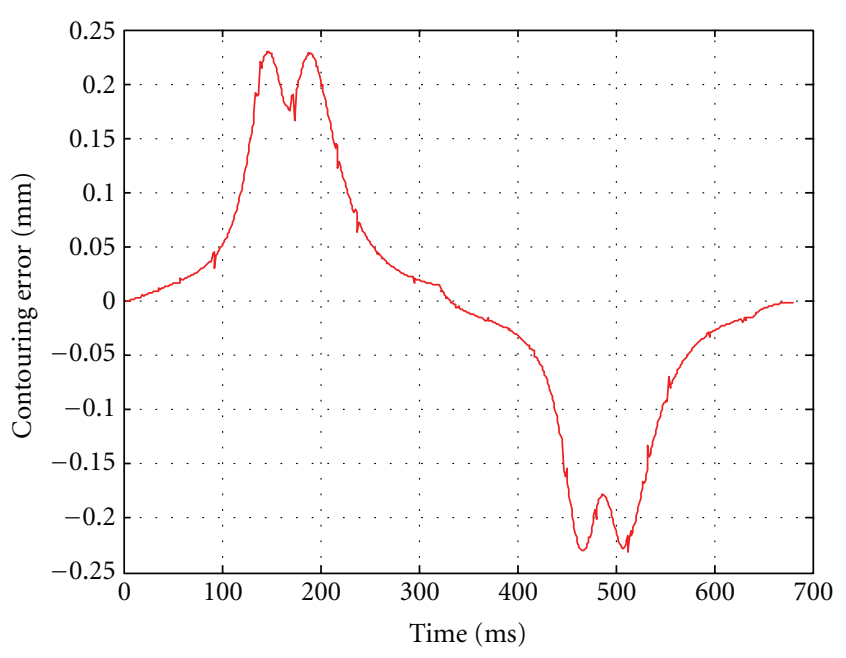

(b)

Figure 19: Contouring error (a) Taylor estimator and (b) $\operatorname{IME~}\left(T_{s}=0.5 \mathrm{~ms}\right.$ and average SDQ $\left.=1.8\right)$.

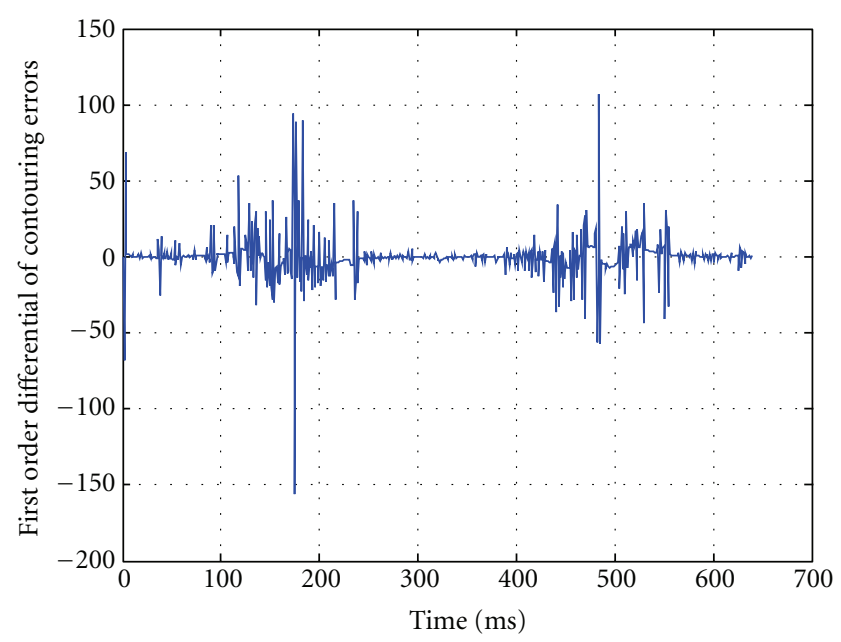

(a)

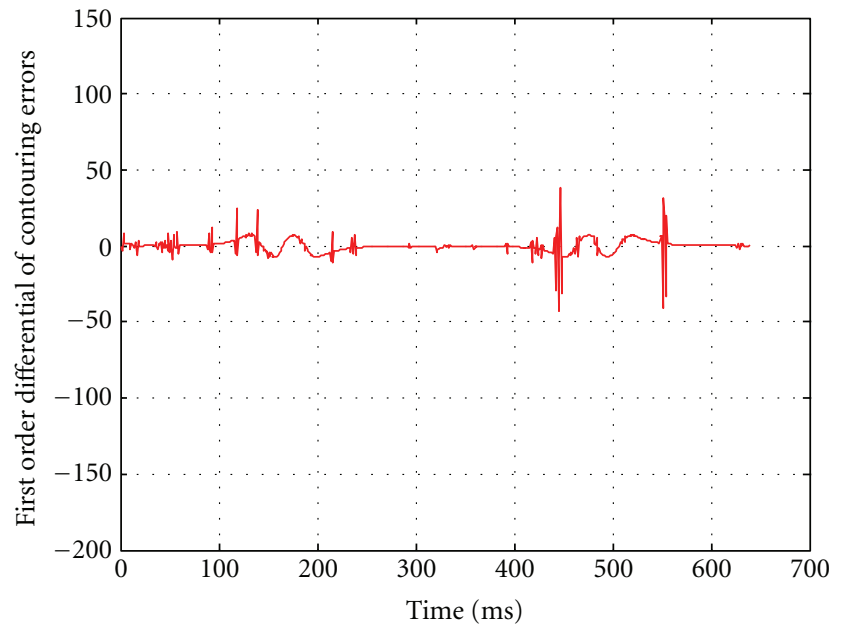

(b)

Figure 20: First-order differential of the contouring errors as shown in Figure 19.

2 and 3. In addition, the 1-delay estimator possesses the best compensation effect when the averaging SDQ is larger than 3. All switching laws according to (14) based on the SDQ thus agree with both the simulation results and the theoretical analysis, as shown in Figure 12. Although the 3rdTaylor estimator has been proven to render more accurate results than the 1-delay estimator [6], Figure 13 further indicates that the proposed intelligent message estimator (IME) renders much better performance under different SDQ, especially as the missing data becomes more serious as shown in Figure 9.

\section{Contouring Accuracy of Motion NCS}

5.1. Simulation Results. Applications of the present estimator based on the SDQ have been applied to the two-axis motion NCS, as shown in Figure 2. The NURBS commands and the system response with averaging SDQ $=0.8$ of the network are shown in Figure 14. Results show that both the 3rd-Taylor estimator and the proposed estimator can reduce the effects of data dropout at a lower averaging SDQ. Figure 15 also shows that the contouring error obtained by applying the proposed estimator is significantly reduced to achieve better contouring accuracy.

Figure 16 shows that the present estimator even renders a much better contouring accuracy when the averaging SDQ increases to 2.6. Furthermore, when the value of the averaging SDQ increases to 3.1, the Taylor estimator will lead to an unstable motion as shown in Figure 17. Under such circumstances, the proposed estimator still results in a stable motion and maintains the contouring accuracy well.

5.2. Experimental Results. The proposed estimator was applied to two Tamagawa motors with NCS realization, as 


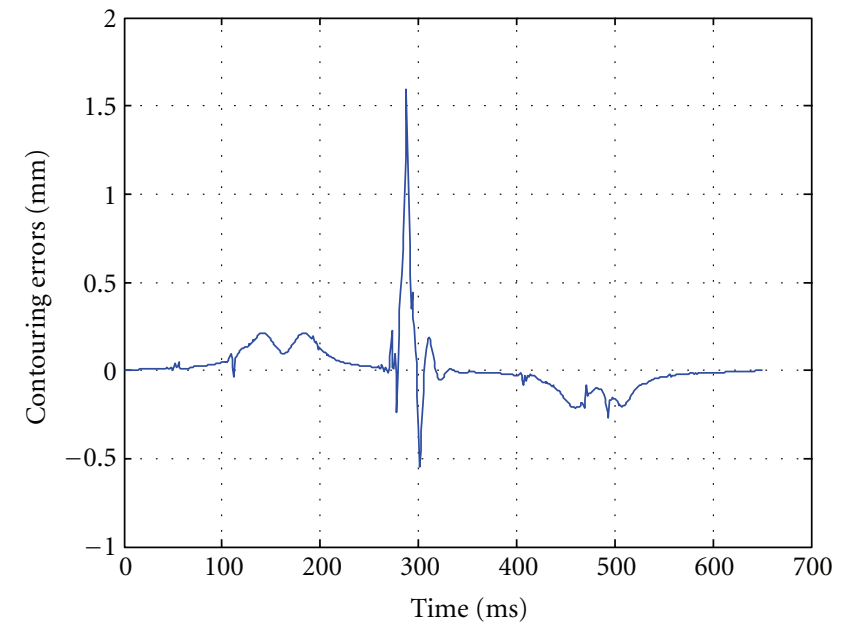

(a)

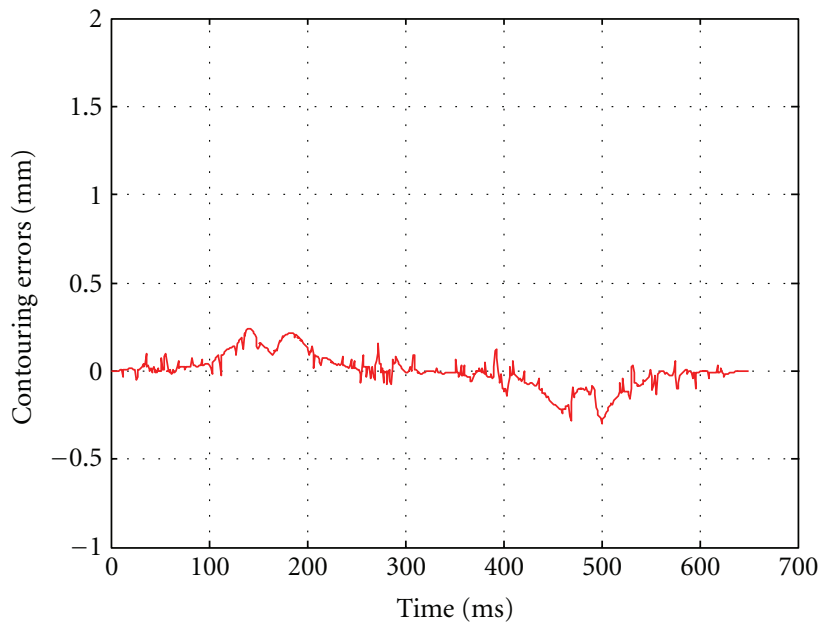

(b)

Figure 21: Contouring error with (a) Taylor estimator and (b) IME $\left(T_{s}=0.4 \mathrm{~ms}\right.$ and average SDQ $\left.=2.7\right)$.

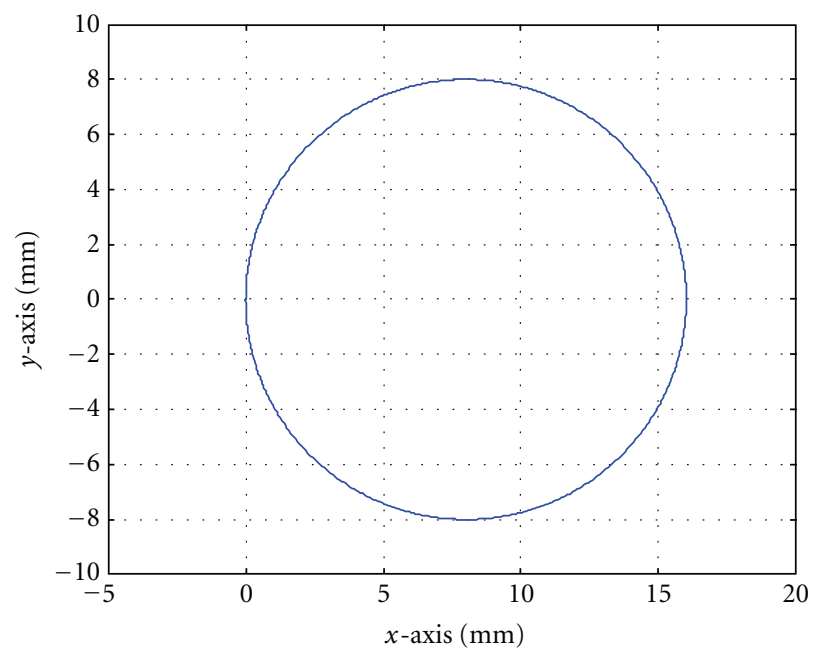

(a)

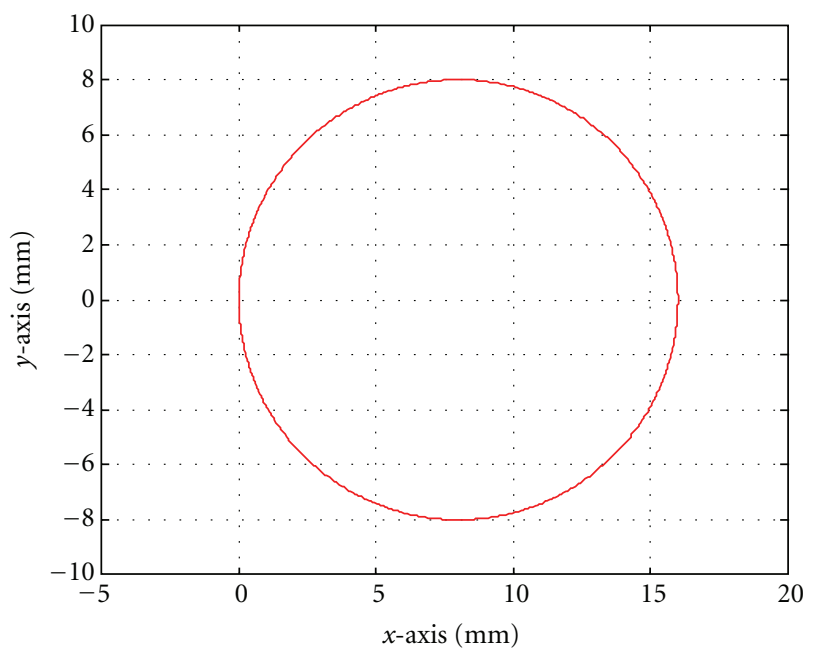

(b)

Figure 22: Circular response of (a) the Taylor estimator and (b) IME $\left(T_{s}=0.5 \mathrm{~ms}\right.$ and average SDQ $\left.=1.8\right)$.

shown in Figure 18. The butterfly NURBS profile for both the $x$-axis and $y$-axis position amplitudes is $30 \mathrm{~mm}$ under the feed rate $3,000 \mathrm{~mm} / \mathrm{min}$ for experiments. Furthermore, the averaging SDQ is measured as 1.8 and 2.7 for the present CAN-bus implementation with the baud rate $1 \mathrm{Mbit} / \mathrm{sec}$ under different sampling periods as $0.5 \mathrm{~ms}$ and $0.4 \mathrm{~ms}$, respectively. Experimental results indicate that increasing the sampling rate will result in more serious missing data due to the saturation of network bandwidth.

Figure 19 also shows the contouring error when the averaging SDQ is 1.8. The first-order differential results of the measured contouring error with less oscillation are also shown in Figure 20. All results indicate that the proposed estimator renders a more stable and reliable motion than the Taylor estimator. By observing the contouring error as shown in Figure 21 with a more serious data dropout (average SDQ 2.7), results also show that the proposed estimator is more effective in reducing the asynchronization effect than the Taylor estimator. Similar results provided by the circular NURBS profile for the motion NCS obtained as shown in Figures 22 and 23 also indicate applicability of the proposed estimator to different motion profiles.

\section{Conclusions}

As the online measured time delay is crucial for the NCS controller design [20], this paper successfully measures transmitted signals with different dropout rates and proposes the SDQ, which is a suitable index for the dropout rate and concentration of missing commands in motion NCS. The missing commands can also be estimated by applying the proper order of LSE. Motion NCS with the proposed estimator render significant improvements in accuracy. 


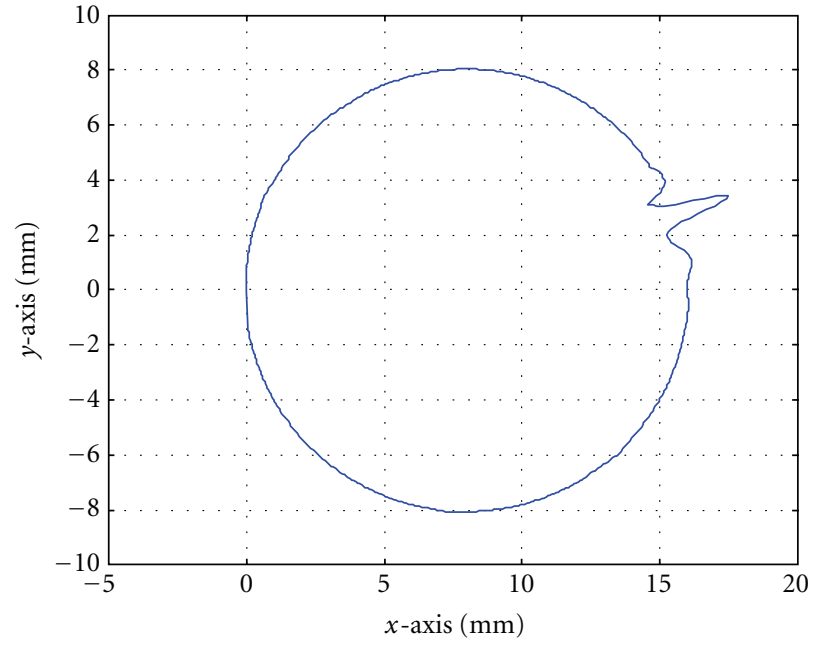

(a)

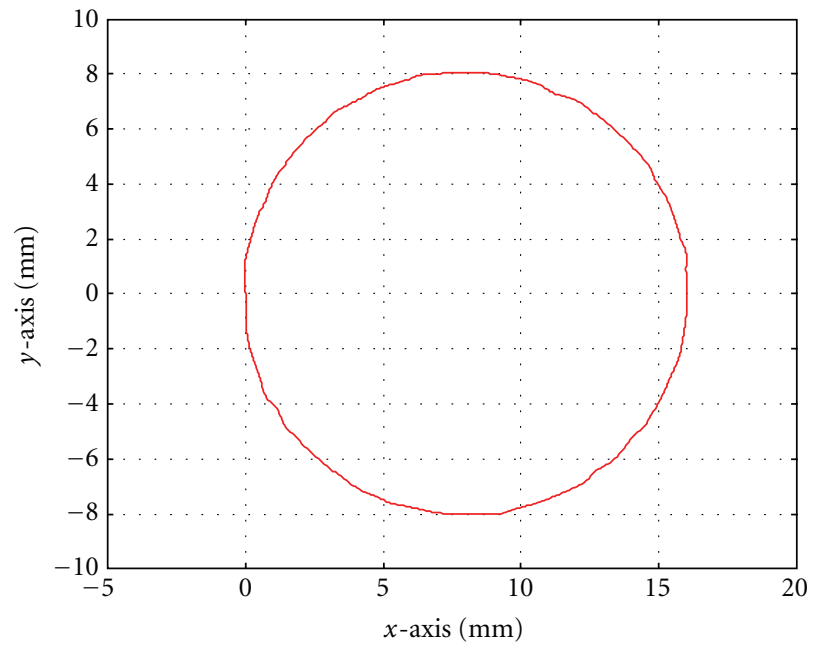

(b)

FIgURE 23: Circular response of (a) Taylor estimator and (b) the proposed estimator $\left(T_{s}=0.4 \mathrm{~ms}\right.$ and average $\left.\mathrm{SDQ}=2.7\right)$.

In a real motion system, commands usually consider position, velocity, and acceleration. Thus, $N=3$ is a suitable order for the least square estimator $\operatorname{LSE}(M, N)$, and 5 is chosen as the window length $M$, with more accurate estimation results if there are no or if there is only one missing data within the window length. A lower order of LSE will be determined if the QoS for the network worsens. The 1-delay estimator can be adopted only for the worst communication cases, as in (14).

The proposed estimator with a switching mechanism based on SDQ leads to the lowest contouring error. Results indicate that with the proposed estimator in motion NCS, contouring accuracy can be maintained even under severe missing commands compared with the 1-delay and the Taylor estimators. Experimental results have also proven the feasibility and applicability of the proposed approach in motion NCS.

\section{Acknowledgment}

This work was supported by the National Science Council, Taiwan, under Grant NSC 98-2218-E-009-005.

\section{References}

[1] S. Zampieri, "Trends in networked control systems," in Proceedings of the 17th World Congress of the International Federation of Automatic Control (IFAC'08), pp. 2886-2894, Seoul, Korea, July 2008.

[2] J. P. Hespanha, P. Naghshtabrizi, and Y. Xu, "A survey of recent results in networked control systems," Proceedings of the IEEE, vol. 95, no. 1, pp. 138-162, 2007.

[3] F. Abdollahi and K. Khorasani, "A novel Ho control strategy for design of a robust dynamic routing algorithm in traffic networks," IEEE Journal on Selected Areas in Communications, vol. 26, no. 4, pp. 706-718, 2008.
[4] T. Matiakis, S. Hirche, and M. Buss, "Control of networked systems using the scattering transformation," IEEE Transactions on Control Systems Technology, vol. 17, no. 1, pp. 60-67, 2009.

[5] G. P. Liu, Y. Xia, J. Chen, D. Rees, and W. Hu, "Networked predictive control of systems with random network delays in both forward and feedback channels," IEEE Transactions on Industrial Electronics, vol. 54, no. 3, pp. 1282-1297, 2007.

[6] C. C. Hsieh, P. L. Hsu, and B. C. Wang, "The motion message estimator in real-time network control systems," in Proceedings of the 32nd Annual Conference on IEEE Industrial Electronics (IECON '06), pp. 4632-4637, November 2006.

[7] C. C. Hsieh, P. L. Hsu, and B. C. Wang, "The motion message estimator in networked control systems," in Proceedings of the 17th World Congress of the International Federation of Automatic Control (IFAC '08), pp. 11606-11611, Seoul, Korea, July 2008.

[8] B. Sinopoli, L. Schenato, M. Franceschetti, K. Poolla, and S. Sastry, "Optimal linear LQG control over lossy networks without packet acknowledgment," Asian Journal of Control, vol. 10, no. 1, pp. 3-13, 2008.

[9] M. Sahebsara, T. Chen, and S. L. Shah, "Optimal $H_{2}$ filtering in networked control systems with multiple packet dropout," IEEE Transactions on Automatic Control, vol. 52, no. 8, pp. 1508-1513, 2007.

[10] S. Sun, L. Xie, W. Xiao, and N. Xiao, "Optimal filtering for systems with multiple packet dropouts," IEEE Transactions on Circuits and Systems II, vol. 55, no. 7, pp. 695-699, 2008.

[11] S. Chai, G. P. Liu, D. Rees, and Y. Xia, "Design and practical implementation of internet-based predictive control of a servo system," IEEE Transactions on Control Systems Technology, vol. 16, no. 1, pp. 158-168, 2008.

[12] Q. Ling and M. D. Lemmon, "Robust performance of soft realtime networked control systems with data dropouts," in Proceedings of the 41st IEEE Conference on Decision and Control, pp. 1225-1230, December 2002.

[13] S. C. Smith and P. Seiler, "Estimation with lossy measurements: jump estimators for jump systems," IEEE Transactions on Automatic Control, vol. 48, no. 12, pp. 2163-2171, 2003. 
[14] Y. C. Tian and D. Levy, "Compensation for control packet dropout in networked control systems," Information Sciences, vol. 178 , no. 5, pp. 1263-1278, 2008.

[15] C. C. Hsieh and P. L. Hsu, "Analysis and applications of the motion message estimator for network control systems," Asian Journal of Control, vol. 10, no. 1, pp. 45-54, 2008.

[16] L. Piegl and W. Tiller, The NURBS Book, Springer, New York, NY, USA, 1995.

[17] M. Gopi and S. Manohar, "A unified architecture for the computation of B-spline curves and surfaces," IEEE Transactions on Parallel and Distributed Systems, vol. 8, no. 12, pp. 1275-1287, 1997.

[18] F. L. Lian, J. R. Moyne, and D. M. Tilbury, "Performance evaluation of control networks: Ethernet, ControlNet, and DeviceNet," IEEE Control Systems Magazine, vol. 21, no. 1, pp. 66-83, 2001.

[19] H. W. Sorenson, "Least-square estimation: from Gauss to Kalman," IEEE Spectrum, vol. 7, no. 7, pp. 63-68, 1970.

[20] C. L. Lai and P. L. Hsu, "Design the remote control system with the time-delay estimator and the adaptive smith predictor," IEEE Transactions on Industrial Informatics, vol. 6, no. 1, pp. 73-80, 2010. 

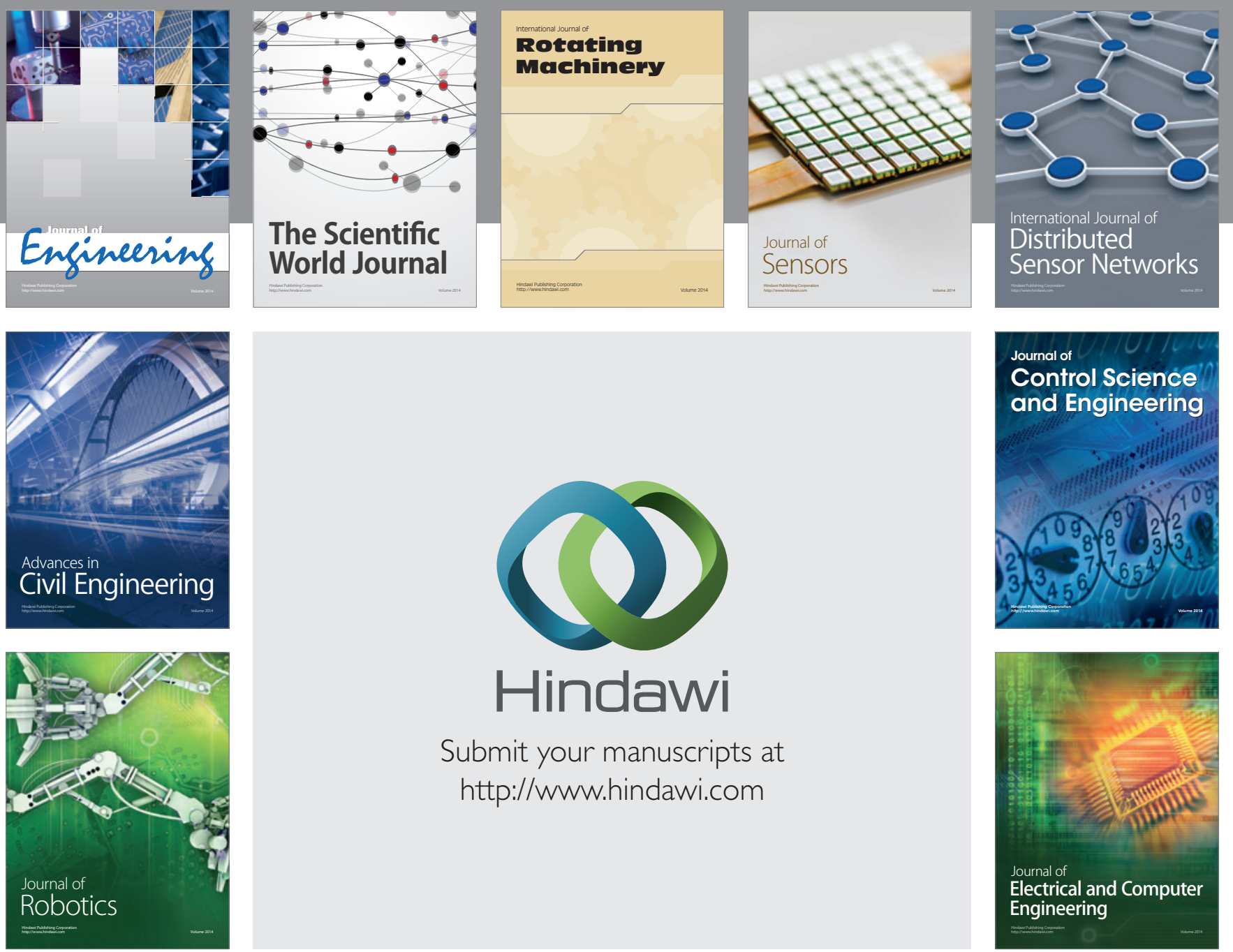

Submit your manuscripts at

http://www.hindawi.com
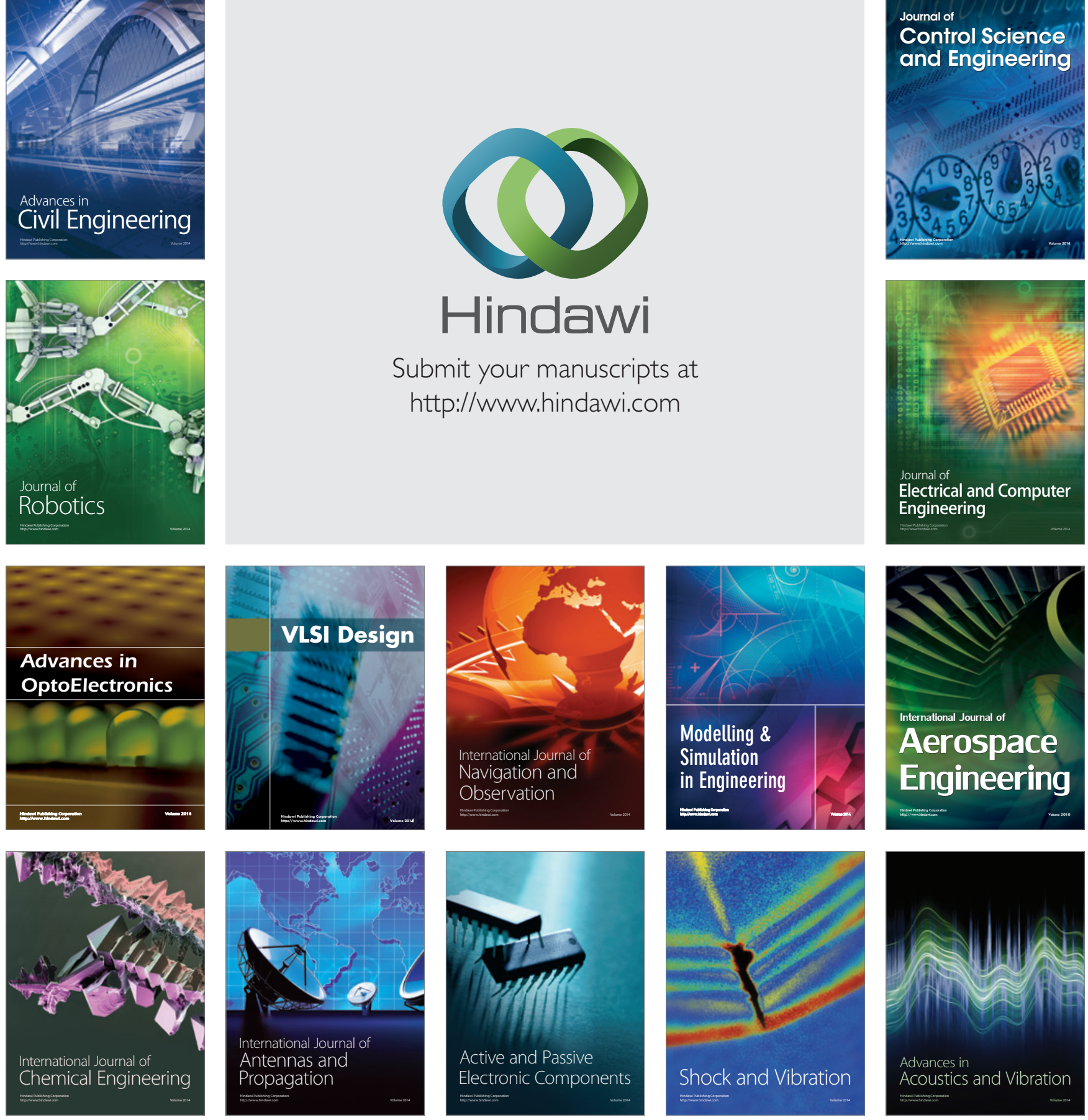